\title{
Thermally driven spin injection from a ferromagnet into a non-magnetic metal
}

\author{
A. Slachter ${ }^{\star}$, F. L. Bakker, J-P. Adam and B. J. van Wees
}

Creation, manipulation and detection of spin-polarized carriers are the key elements of spin-based electronics ${ }^{1,2}$. Most practical devices $^{3-5}$ use a perpendicular geometry in which the spin currents are accompanied by charge currents. In recent years, new sources of pure spin currents (that is, transport of spin angular momentum without charge currents) have been demonstrated ${ }^{6-9}$ and applied ${ }^{10-12}$. Here we demonstrate a conceptually new source of pure spin current driven by the flow of heat across the interface between a ferromagnet and a non-magnetic metal. This spin current is generated because, in a ferromagnet, the Seebeck effect-which describes the generation of a voltage as a result of a temperature gradient-is spin dependent ${ }^{13,14}$. We studied this new source of spin currents experimentally in a non-local lateral geometry and developed a three-dimensional model that describes the heat, charge and spin transport in this geometry, enabling us to quantify this process ${ }^{15}$. We obtain a spin-dependent Seebeck coefficient for Permalloy of $-3.8 \mu \mathrm{VK}^{-1}$, suggesting that thermally driven spin injection is a feasible alternative for electrical spin injection in, for example, spin-transfer-torque experiments ${ }^{16}$.

The interplay of spin-dependent conductivity and thermoelectricity has been known since half a century ago, when it was used to describe the conventional Seebeck effect of ferromagnetic metals ${ }^{17}$. The discovery of the giant magnetoresistance effect ${ }^{3}$ sparked the interest of the community in spin-dependent conductivity and new spin electronics that still exists today ${ }^{4,5,8,18}$. Owing to experimental difficulties in controlling heat flows it was only very recently that thermoelectric spintronics was investigated ${ }^{19,20}$, leading to the new field of spin caloritronics ${ }^{13}$. A relevant example is given in ref. 9, which interprets its results in terms of the generation of a bulk spin accumulation due to an applied temperature gradient in a ferromagnetic film. In contrast, the effect we describe in this paper arises from a heat current flowing through a ferromagnetic/non-magnetic metal (FM/NM) junction, which creates a spin accumulation at the interface.

The concept of how we generate a heat current over an FM/NM junction and subsequently measure the spin accumulation is shown in Fig. 1. The scheme is essentially a lateral non-local spin-valve structure $^{6}$ with the electrical injection replaced by thermal spin injection. We use this non-local scheme to separate spin injection from possible spurious effects ${ }^{6,11,12}$ and because the observed thermally generated non-spin-related voltage, to which we refer as the baseline resistance, enables us to extract the temperature distribution in the device by comparing this with modelling ${ }^{15}$.

We first formulate an appropriate diffusive-transport theory for thermally driven spin injection. The Seebeck coefficient indicates that an applied temperature gradient across a conductor generates an electric field ${ }^{21}$. In a ferromagnet, the transport processes for the majority and minority spins are different, leading to

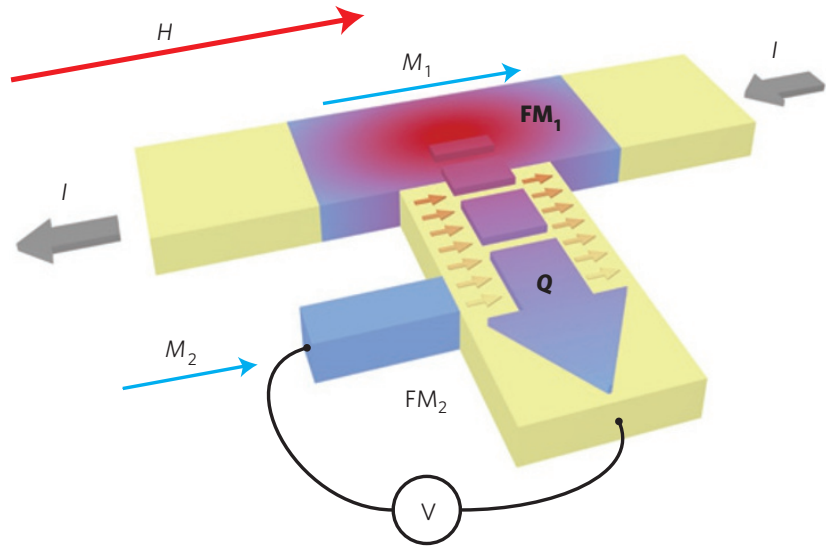

Figure 1 | Conceptual diagram. A charge current $I$ is sent through ferromagnet $1\left(F M_{1}\right)$ causing Joule heating owing to the large resistivity of $\mathrm{FM}_{1}$. The NM contacts (yellow) are highly thermally conductive, thereby providing heat sinks. The heat current $Q$ through the centre $F M_{1} / N M$ interface injects a spin current into the NM depending on the magnetization direction $M_{1}$. The generated spins diffuse towards the $F M_{2} / N M$ interface, where they generate a potential $\Delta \mu=P \mu_{\mathrm{s}}$ depending on the magnetization direction $M_{2}$. As a consequence of Joule heating, the signal expected to arise from thermal spin injection scales with $\nabla T \propto R^{2}$. This potential is measured using the indicated voltage scheme by selectively switching the magnetization directions $M_{1}$ and $M_{2}$ by a magnetic field $H$.

a spin-dependent conductivity $\sigma_{\uparrow, \downarrow}$ and Seebeck coefficient $S_{\uparrow, \downarrow}$ (refs 14,17). The former is used to describe magnetoelectronics ${ }^{22}$ in FM/NM systems, where the latter is usually disregarded. To consider what happens when heat is sent through the system, we write the spin-dependent currents in both the bulk FM and NM regions:

$$
J_{\uparrow, \downarrow}=-\sigma_{\uparrow, \downarrow}\left(\frac{1}{e} \nabla \mu_{\uparrow, \downarrow}+S_{\uparrow, \downarrow} \nabla T\right)
$$

here $\mu_{\uparrow, \downarrow}$ is the spin-dependent chemical potential and $T$ is the temperature. When a heat current $Q$ is sent through the bulk of a ferromagnet in the absence of a charge current, a spin current $J_{\mathrm{s}}=J_{\uparrow}-J_{\downarrow}=-\sigma_{\mathrm{F}}\left(1-P^{2}\right) S_{\mathrm{s}} \nabla T / 2$ flows driven by the spin-dependent Seebeck coefficient, which we define as $S_{s} \equiv S_{\uparrow}-S_{\downarrow}$ (Supplementary A). Here $P$ is the conductivity polarization $P=\left(\sigma_{\uparrow}-\sigma_{\downarrow}\right) /\left(\sigma_{\uparrow}+\sigma_{\downarrow}\right)$ of the ferromagnet and $\sigma_{\mathrm{F}}$ is the conductivity of the ferromagnet. We note that our definition of $S_{\mathrm{s}}$ should be distinguished from the spin Seebeck coefficient defined in ref. 9, where a so-called 'entropy term' was included ${ }^{23}$. We do not include this term in our analysis. 


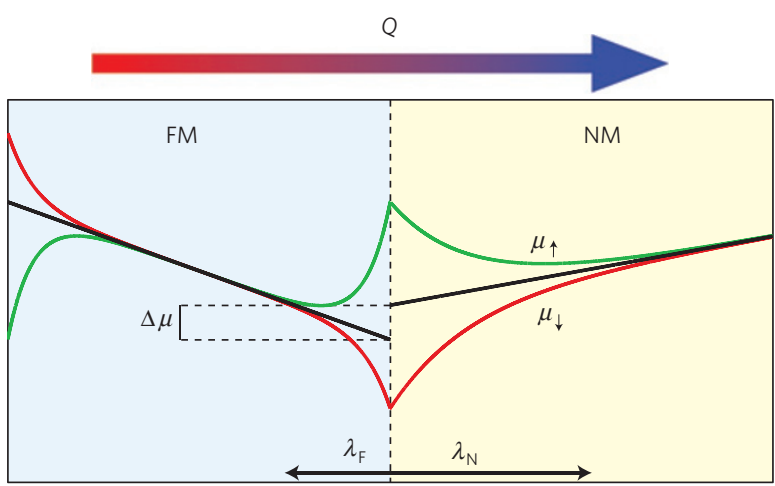

Figure 2 | Thermal spin injection by the spin-dependent Seebeck coefficient across an FM/NM interface. Schematic figure showing the resulting spin-dependent chemical potentials $\mu_{\uparrow, \downarrow}$ across an FM/NM interface when a heat current $Q=-k \nabla T$ crosses it. The heat current is taken to be continuous across the interface, leading to a discontinuity in $\nabla T$. No currents are allowed to leave the FM; nevertheless, a spin current proportional to the spin-dependent Seebeck coefficient flows through the bulk FM, which needs to become unpolarized in the bulk NM. This creates a spin imbalance $\mu_{\uparrow}-\mu_{\downarrow}$ at the boundary, which relaxes in the FM and NM on the length scale of their respective spin-relaxation lengths $\lambda_{i}$. A thermoelectric interface potential $\Delta \mu=P \mu_{\mathrm{s}}$ also builds up. On the left side no spin current is allowed to leave, leading to a spin accumulation of opposite sign.

To quantify the thermal injection of spins we consider the $\mathrm{FM} / \mathrm{NM}$ interface and solve the Valet-Fert equation ${ }^{24}$ in a fashion similar as in ref. 25 (Supplementary A). The result is depicted in Fig. 2. A spin accumulation $\mu_{\mathrm{s}}=\mu_{\uparrow}-\mu_{\downarrow}$ appears at the interface driven by the abrupt change of spin current going from the bulk FM to the bulk NM, thereby acting as an effective source of spins at the interface. The resulting spin accumulation has the following expression:

$$
\frac{\mu_{\mathrm{s}}}{\nabla T_{\mathrm{FM}}}=-e \lambda_{\mathrm{F}} S_{\mathrm{s}} R_{\mathrm{mis}}
$$

where $R_{\text {mis }}=R_{\mathrm{N}} /\left(R_{\mathrm{N}}+R_{\mathrm{F}} /\left(1-P^{2}\right)\right)$ is a conductivity mismatch ${ }^{26}$ factor in which $R_{\mathrm{F}}=\lambda_{\mathrm{F}} / \sigma_{\mathrm{F}}$ and $R_{\mathrm{N}}=\lambda_{\mathrm{N}} / \sigma_{\mathrm{N}}$ are the spin resistances determined by the relaxation lengths $\lambda_{\mathrm{F}}$ and $\lambda_{\mathrm{N}}$ and the conductivities $\sigma_{\mathrm{F}}$ and $\sigma_{\mathrm{N}}$. For the metallic interfaces under consideration in this paper, this factor is very close to unity. The resulting spin accumulation induced by the heat flow $Q=-k_{\mathrm{FM}} \nabla T_{\mathrm{FM}}$, where $k_{\mathrm{FM}}$ is the thermal conductivity of and $\nabla T_{\mathrm{FM}}$ the thermal gradient in the ferromagnet, is determined solely by the spin-dependent Seebeck coefficient $S_{\mathrm{s}}$ and the ferromagnetic spin relaxation length $\lambda_{\mathrm{F}}$. Its direction is determined by the sign of the spin-dependent Seebeck coefficient, which changes sign when the magnetization of the ferromagnet reverses.

The signal due to thermally driven spin injection in the geometry of Fig. 1 scales with Joule heating: $\nabla T \propto I^{2}$, where $I$ is the charge current sent through the device. Therefore, we use a lock-in technique to determine the relevant parameters $R_{1}\left(\mu \mathrm{V} \mathrm{mA}^{-1}\right)$ and $R_{2}\left(\mu \mathrm{V} \mathrm{mA}^{-2}\right)$ from the observed voltage ${ }^{15}$ :

$$
V=R_{1} I+R_{2} I^{2}+\cdots
$$

The baseline 'resistance', defined in terms of a parallel and an antiparallel contribution as $\left(R_{i}{ }^{\mathrm{P}}+R_{i}{ }^{\mathrm{AP}}\right) / 2=R_{i}{ }^{\mathrm{b}}$, enables us to extract the magnitude of Joule and Peltier heating effects and possible conventional Ohmic potential drops ${ }^{15}$. Here $R_{1}{ }^{\mathrm{b}}$ is determined by the Ohmic potential drop and Peltier heating/cooling measured by the $\mathrm{FM}_{2}-\mathrm{NM}$ thermocouple, whereas the baseline resistance $R_{2}{ }^{\mathrm{b}}$ is

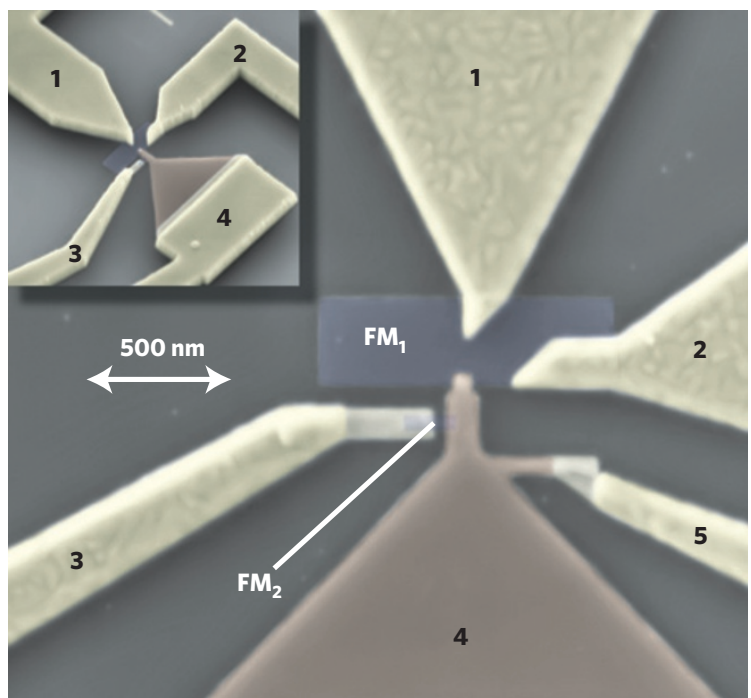

Figure 3 | Coloured SEM picture of the fabricated device. The device consists of two 15 -nm-thick Permalloy $\left(\mathrm{Ni}_{80} \mathrm{Fe}_{20}\right)$ ferromagnets, $\mathrm{FM}_{1}$ and $\mathrm{FM}_{2}$, of dimensions $1 \mu \mathrm{m} \times 300 \mathrm{~nm}$ and $150 \times 40 \mathrm{~nm}^{2}$, separated from each other by $100 \mathrm{~nm}$. They are connected by a $60-\mathrm{nm}$-thick copper funnel with small effective FM/NM contact areas of $40 \times 40 \mathrm{~nm}^{2}$ and $30 \times 40 \mathrm{~nm}^{2}$. 5/175-nm-thick Ti/Au Contacts 1 and 2 are placed asymmetrically on $\mathrm{FM}_{1}$ to Joule heat it, whereas Contacts 3 and 4 are used to measure Joule heating and thermal spin injection. An extra Contact 5 is present to measure a regular non-local spin-valve signal.

determined by Joule heating measured by the same thermocouple. The spin-dependent contribution $R_{i}{ }^{\mathrm{P}}-R_{i}{ }^{\mathrm{AP}}=R_{i}{ }^{\text {s }}$ to $R_{1}$ is due to a conventional spin-valve signal, whereas this contribution to $R_{2}$ comes from thermal spin injection.

A dedicated device was fabricated to study this effect and is shown in Fig. 3. The heating of $\mathrm{FM}_{1}$ has been kept very localized to an area of $150 \times 150 \mathrm{~nm}^{2}$ by using thick gold contacts. Moreover, the contacts are placed asymmetrically to minimize the possible current flowing in and out of the $\mathrm{FM}_{1} / \mathrm{NM}$ interface. An extra Contact 5 is present to be able to send a current directly through the $\mathrm{FM}_{1} / \mathrm{NM}$ interface. By comparing the obtained signal $R_{1}{ }^{\mathrm{s}}$ with a model (see Methods), we can extract the spin injection/detection efficiency ${ }^{15}$, which has been made as high as possible by keeping the size of the FM/NM contacts small. All measurements are made at room temperature.

Figure 4 shows our principal results on thermal spin injection. Four distinct $\mathrm{P}-\mathrm{AP}$ and $\mathrm{AP}-\mathrm{P}$ switches are observed, up to $70 \mathrm{nV}$ in magnitude, scaling with $I^{2}$ on a large background originating from the $\mathrm{Py}_{2} / \mathrm{Cu}$ thermocouple.

The interpretation of the obtained signals requires a detailed knowledge of the heat, charge and spin currents in the device. For this purpose a three-dimensional thermoelectric spin model was constructed, which extends the spin-dependent current model ${ }^{24}$ to include thermoelectricity as well as thermal spin injection by the spin-dependent Seebeck coefficient.

The calculated average contribution $R_{2}{ }^{\mathrm{b}}$ is $2.4 \mu \mathrm{VmA} \mathrm{m}^{-2}$ lower than the observed $7.69 \mu \mathrm{V} \mathrm{mA}^{-2}$. A difference between the observed and modelled values has been seen before in non-local spin-valve samples ${ }^{15}$. It can be explained by a reduction in the Permalloy thickness due to its oxidation, which effectively increases the Joule heating. In the following, we scale the overall Joule heating in our model to fit our measured result $R_{2}{ }^{\mathrm{b}}$. We then find that we are able to heat $\mathrm{FM}_{1}$ to a maximum of $\approx 40 \mathrm{~K}$, at which $\nabla T_{\mathrm{FM}}$ at the

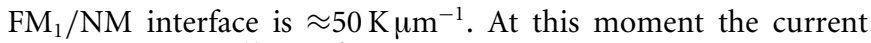
density is $\approx 8 \times 10^{11} \mathrm{~A} \mathrm{~m}^{-2}$, close to the point where the device will fail owing to electromigration. 


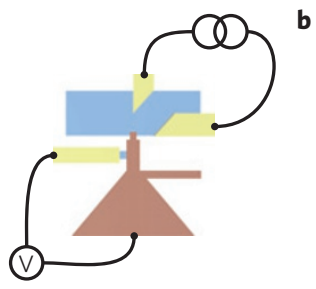

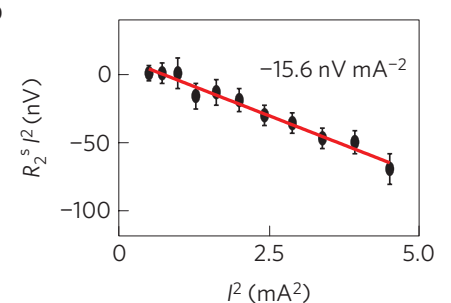

$12\left(m A^{2}\right)$

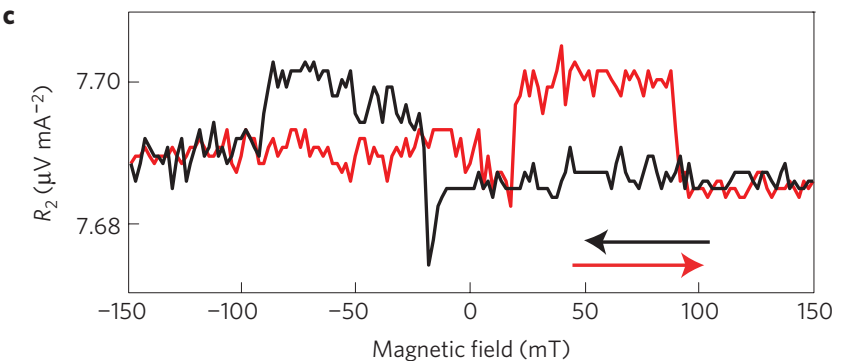

Figure 4 | Thermal-spin-injection measurements. a, Measurement scheme of the experiment. $\mathbf{b}$, Second-harmonic measurement result $R_{2} \mathrm{~s} I^{2}$ $(\mathrm{nV})$ of the observed thermal spin signal as a function of $R^{2}$. The error bars represent the standard deviation in the average height of the four P-AP and AP-P switches. c, Measured second-harmonic signal at a root mean squared current of $1.5 \mathrm{~mA}$, showing the four distinct switches resulting from the magnetization alignment of $\mathrm{FM}_{1}$ and $\mathrm{FM}_{2}$ illustrating thermal spin injection.

Electrical spin injection was also measured by sending the current directly through the $\mathrm{Py}_{1} / \mathrm{Cu}$ interface, and the result is shown in Fig. $5 c$. From the measured resistance $R_{\text {NLSV }}(\Omega)$, we see that a relatively large $9 \mathrm{~m} \Omega$ spin-valve signal is present on top of a $1.05 \Omega$ background, which is only slightly different to the $7.8 \mathrm{~m} \Omega$ and $640 \mathrm{~m} \Omega$ calculated signals with the metallic spin parameters $\lambda_{\mathrm{Cu}}=350 \mathrm{~nm}, \lambda_{\mathrm{Py}}=5 \mathrm{~nm}$ and $P_{\mathrm{Py}}=0.25$ obtained from previously fabricated samples ${ }^{6,15}$. Here $P_{\mathrm{Py}}$ is positive, as shown before ${ }^{27}$.

The observed thermal-spin-injection signal $R_{2}{ }^{\mathrm{s}}=-15.6 \mathrm{nV} \mathrm{mA}^{-2}$ is determined from Fig. $4 \mathrm{~b}$. We obtain a spin-dependent Seebeck coefficient for Permalloy of $-3.8 \mu \mathrm{VK}^{-1}$, a fraction of the conventional Seebeck coefficient $S_{\mathrm{F}}=-20 \mu \mathrm{V} \mathrm{K}^{-1}$ (ref. 9). This gives a polarization of the Seebeck coefficient of $P_{\mathrm{S}}=S_{\mathrm{s}} / S_{\mathrm{F}}=0.19$, not too different from the spin polarization of the conductivity. At the maximum currents used, we extract a net spin accumulation of $\approx 1 \mu \mathrm{eV}$ at the $\mathrm{FM}_{1} / \mathrm{NM}$ interface. The magnitude of the spin-dependent Seebeck coefficient is in good agreement with theoretical predictions ${ }^{14,16}$. The previously deduced spin Seebeck coefficient $S_{\mathrm{s}}=-2 \mathrm{nV} \mathrm{K}^{-1}$ and the spin-dependent Seebeck coefficient in this paper are similar by definition, but describe different physical processes (Supplementary A). The discrepancy between the two values arises from the modelling, which is different. We also do not exclude that the relevant physics itself in their experiment could be different, as alternative explanations have been reported ${ }^{28}$.

In addition to the thermal-spin-injection signal, a small regular spin-valve signal $R_{1}{ }^{\mathrm{s}}=-20 \mu \Omega$ is also present and is shown in Fig. 5a. The baseline resistance $R_{1}{ }^{\mathrm{b}}$ of $90 \mu \Omega$ is in line with the calculated $95 \mu \Omega$. This is caused by Peltier heating and cooling of the two current-injecting contacts ${ }^{15}$.

The negative regular spin-valve signal $R_{1}{ }^{\text {s }}$ can be understood as follows. Owing to the high conductivity of the copper, a fraction of the current flows into and out of the $\mathrm{Py}_{1} / \mathrm{Cu}$ interface electrically injecting spins. A small net spin accumulation at the detector interface remains, caused by the asymmetric placement of $\mathrm{FM}_{2}$. This is illustrated by the calculation of the spin accumulation at the $\mathrm{Py}_{1} / \mathrm{Cu}$ interface shown in Fig. 5d, which shows the high

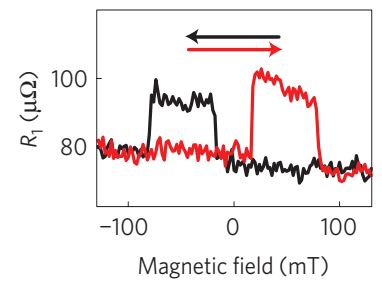

c
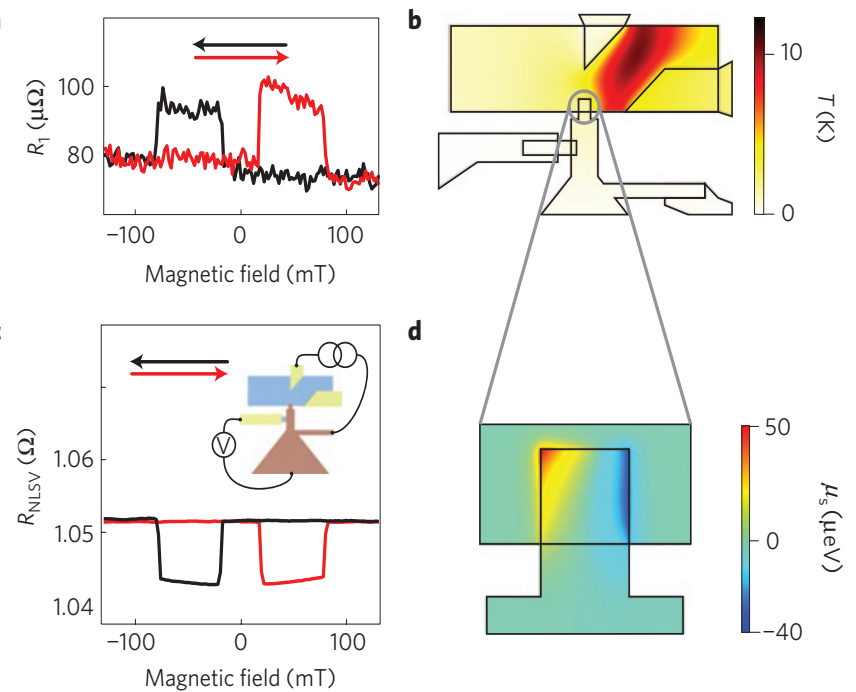

Figure 5 | Rectification effects and electrical spin injection.

a, First-harmonic measurement $R_{1}$ for the measurement set-up of Fig. 4 . b. Calculated temperature distribution at a height of $10 \mathrm{~nm}$ with a current of $2 \mathrm{~mA}$ sent through $\mathrm{FM}_{1}$. This illustrates the localized Joule heating, Peltier cooling and heating of the two Au/Py current-injecting contacts and subsequent thermal conduction towards the three connected metallic contacts. c, Measured electrical spin-injection scheme and resulting spin valve. d, Calculated spin accumulation at a height of $10 \mathrm{~nm}$. A small part of the current path is short-circuited by the Cu connection so that $4 \%$ still flows in and out of the $\mathrm{Py}_{1} / \mathrm{Cu}$ contact because of its large conductivity. This creates a large positive and negative spin accumulation. Owing to the asymmetry in spin injection and the asymmetrical placement at $\mathrm{FM}_{2}$, a small fraction of $3 \%$ is still predicted to give a small regular spin-valve signal $R_{1}{ }^{\mathrm{s}}$.

geometrical dependence of this effect. The observed $R_{1}{ }^{\mathrm{s}}$ is somewhat smaller than the calculated $-45 \mu \Omega$. We believe that the small $40 \times 40 \mathrm{~nm}^{2}$ size of the copper contact makes sure copper grain size, lithographic precision and ballistic effects start dominating the magnitude of this effect.

A previous device showed a thermal-spin-injection signal of $-5 \mathrm{nV} \mathrm{mA}^{-2}$ at an FM-FM distance of $400 \mathrm{~nm}$, only visible at the highest current (Supplementary B). A similar calculation gives $S_{\mathrm{s}}$ Py $=-5 \mu \mathrm{VK}^{-1}$, in agreement with the analysis for the sample presented. This measurement also rules out any influence of nonlinear behaviour of the process seen in the $R_{1}{ }^{\mathrm{s}} I$ signal on the $R_{1}{ }^{\mathrm{s}} I^{2}$ signal (Supplementary C).

Now that the parameters governing equation (2) are known, we may compare this to the electrical-spin-injection results for the transparent $\mathrm{Py} / \mathrm{Cu}$ interface. We can calculate that $\mu_{\mathrm{S}} / \nabla T \approx 2 \times 10^{-14} \mathrm{eV} \mathrm{m} \mathrm{K}^{-1}$ for thermal spin injection versus $\mu_{\mathrm{S}} / J \approx 3 \times 10^{-16} \mathrm{eV} \mathrm{m}^{2} \mathrm{~A}^{-1}$ for electrical spin injection through a transparent $\mathrm{Cu} / \mathrm{Py}$ contact.

Owing to the lateral non-local geometry and Joule-heating method used in this paper, we are limited to a maximum


perpendicular geometry switching by spin-transfer torque $e^{5}$ this does not have to be the case. To switch the magnetization by electrical spin-transfer torque ${ }^{5}$ we need a typical charge current density of $\approx 5 \times 10^{11} \mathrm{~A} \mathrm{~m}^{-2}$. The same stack should be able to switch by applying a temperature difference of only a few tens of degrees as earlier theoretical ${ }^{14,16}$ and experimental ${ }^{29}$ studies have indicated. This simple example shows that, despite the weak signals observed in this paper, thermal spin injection can be a viable alternative, or even work alongside, electrical spin injection. 


\section{Methods}

Fabrication. The sample in this paper was fabricated by a one-step optical and five-step electron beam lithography process. In each step, metals are deposited using e-beam deposition. For the e-beam lithography process a PMMA $950 \mathrm{~K}$ resist is used of 70-400 nm thickness depending on the thickness of the deposited material and resilience to Ar ion milling. The first e-beam lithography process produces 5/30-nm-thick and 100-nm-wide Ti/Au markers, which using an automatic alignment procedure can be aligned to in the next e-beam deposition steps with high precision. In the next four steps, the $15 \mathrm{~nm} \mathrm{Py,} \mathrm{5/30} \mathrm{nm} \mathrm{Ti/Au,}$ $5 / 180 \mathrm{~nm} \mathrm{Ti} / \mathrm{Au}$ and $65 \mathrm{~nm} \mathrm{Cu}$ layers are deposited. For the last three steps, $\mathrm{Ar}$ ion milling was used before deposition to remove any polymer residue and the Py oxide to obtain our highly ohmic contacts.

Measurements. The measurements were made using an a.c. current source of a frequency $<1 \mathrm{kHz}$ far below the characteristic thermoelectric timescale of such sized systems of $\approx 1-100 \mathrm{~ns}$. The obtained signal is sent to three lock-in systems measuring the first, second and third harmonic responses simultaneously. Care was taken in deriving $R_{1}$ and $R_{2}$ by scanning the current from $500 \mu \mathrm{A}$ to $1.5 \mathrm{~mA}$ (root mean squared) to make sure that higher harmonics, as well as cross-talk, were negligible.

Modelling. We constructed a three-dimensional model of the fabricated sample using the finite-element program Comsol Multiphysics. The physics is defined in terms of a thermoelectric spin model where the spin-up, down and heat currents are given by:

$$
\left(\begin{array}{c}
\mathbf{J}_{\uparrow} \\
\mathbf{J}_{\downarrow} \\
\mathbf{Q}
\end{array}\right)=-\left(\begin{array}{ccc}
\sigma_{\uparrow} & 0 & \sigma_{\uparrow} S_{\uparrow} \\
0 & \sigma_{\downarrow} & \sigma_{\downarrow} S_{\downarrow} \\
\sigma_{\uparrow} \Pi_{\uparrow} & \sigma_{\downarrow} \Pi_{\downarrow} & k
\end{array}\right) \cdot\left(\begin{array}{c}
\nabla \mu_{\uparrow} / e \\
\nabla \mu_{\downarrow} / e \\
\nabla T
\end{array}\right)
$$

where $\Pi_{\uparrow, \downarrow}$ are the spin-dependent Peltier coefficients given by $S_{\uparrow, \downarrow} \cdot T_{0}$. Here $T_{0}=300 \mathrm{~K}$, which is the reference temperature of the device. We take these currents to be continuous across boundaries. At the end of all contacts we set the temperature to be $T_{0}$. At Contact 1 in Fig. 3 we set $J_{\uparrow, \downarrow}=J / 2$ to inject a charge current, which is sent through the system by setting $\mu_{\uparrow, \downarrow}=0$ at Contact 2 or 5 . At all other interfaces the currents are set to zero. We include Valet-Fert spin relaxation by assuming $\nabla J_{\uparrow, \downarrow}=\mp\left(1-P^{2}\right) \sigma_{i} / 4 e \lambda_{i}{ }^{2}\left(\mu_{\uparrow}-\mu_{\downarrow}\right)$ in the bulk. Joule heating is included by assuming $\nabla Q=\zeta\left(\mathbf{J}_{\uparrow}{ }^{2} / \sigma_{\uparrow}+\mathbf{J}_{\downarrow}{ }^{2} / \sigma_{\downarrow}\right)$, where a scaling factor $\zeta=3.2$ is used to make the model correspond to the measured $R_{2}{ }^{\mathrm{b}}$. The system was meshed most accurately at the FM/NM interfaces where the mesh size was $1 \mathrm{~nm}$ to accurately calculate thermal spin injection. The dependencies $R_{1}{ }^{(s)}$ up to $R_{4}{ }^{(\mathrm{s})}$ were determined by calculating the results at \pm 1 and $2 \mathrm{~mA}$ for the parallel and antiparallel configurations. The measured resistivities $\sigma_{\mathrm{Au}}=2.2 \times 10^{7} \mathrm{~S} \mathrm{~m}^{-1}$ $\sigma_{\mathrm{Cu}}=4.26 \times 10^{7} \mathrm{~S} \mathrm{~m}^{-1}$ and $\sigma_{\mathrm{Py}}=4.32 \times 10^{6} \mathrm{~S} \mathrm{~m}^{-1}$ were taken as inputs for the model. In this model, the substrate was also taken into account ${ }^{15}$. The Seebeck coefficients $S_{\mathrm{Au}}=1.7 \mu \mathrm{V} \mathrm{K}^{-1}, S_{\mathrm{Cu}}=1.6 \mu \mathrm{V} \mathrm{K}^{-1}$ and $S_{\mathrm{Py}}=-20 \mu \mathrm{V} \mathrm{K}^{-1}$ and thermal conductances $k_{\mathrm{Au}}=300 \mathrm{~W} \mathrm{~m}^{-1} \mathrm{~K}^{-1}, k_{\mathrm{Cu}}=300 \mathrm{~W} \mathrm{~m}^{-1} \mathrm{~K}^{-1}, k_{\mathrm{Py}}=30 \mathrm{~W} \mathrm{~m}^{-1} \mathrm{~K}^{-1}$ and $k_{\text {substrate }}=1 \mathrm{~W} \mathrm{~m}^{-1} \mathrm{~K}^{-1}$ were taken from various sources in the literature ${ }^{9,30}$.

Received 12 April 2010; accepted 6 August 2010; published online 19 September 2010

\section{References}

1. Zutic, I., Fabian, J. \& Das Sarma, S. Spintronics: Fundamentals and applications. Rev. Mod. Phys. 76, 323-410 (2004).

2. Chappert, C., Fert, A. \& Nguyen van Dau, F. The emergence of spin electronics in data storage. Nature Mater. 6, 813-823 (2007).

3. Baibich, M. N. et al. Giant magnetoresistance of $(001) \mathrm{Fe} /(001) \mathrm{Cr}$ magnetic superlattices. Phys. Rev. Lett. 61, 2472-2475 (1988).

4. Kiselev, S. I. et al. Microwave oscillations of a nanomagnet driven by a spin-polarized current. Nature 425, 380-383 (2003).

5. Albert, F. J., Katine, J. A., Buhrman, R. A. \& Ralph, D. C. Spin-polarized current switching of a Co thin film nanomagnet. Appl. Phys. Lett. 77, 3809-3811 (2000)

6. Jedema, F. J., Filip, A. \& van Wees, B. J. Electrical spin injection and accumulation at room temperature in an all-metal mesoscopic spin valve. Nature 410, 345-348 (2001).

7. Valenzuela, S. O. \& Tinkham, M. Direct electronic measurement of the spin Hall effect. Nature 442, 176-179 (2006).

8. Costache, M. V., Sladkov, M., Watts, S. M., van der Wal, C. H. \& van Wees, B. J. Electrical detection of spin pumping due to the precessing magnetization of a single ferromagnet. Phys. Rev. Lett. 97, 216603 (2006).
9. Uchida, K. et al. Observation of the spin Seebeck effect. Nature 455, 778-781 (2008)

10. Yang, T., Kimura, T. \& Otani, Y. Giant spin-accumulation signal and pure spin-current-induced reversible magnetization switching. Nature Phys. 4, $851-854$ (2008)

11. Lou, X. et al. Electrical detection of spin transport in lateral ferromagnet semiconductor devices. Nature Phys. 3, 197-202 (2007).

12. Dash, S. P., Sharma, S., Patel, R. S., de Jong, M. P. \& Jansen, R. Electrical creation of spin polarization in silicon at room temperature. Nature 462, 491-494 (2009).

13. Bauer, G. E., MacDonald, A. H. \& Maekawa, S. Spin caloritronics. Solid State Commun. 150, 459-460 (2010).

14. Tulapurkar, A. A. \& Suzuki, Y. Contribution of electron-magnon scattering to the spin-dependent Seebeck effect in a ferromagnet. Solid State Commun. 150, 466-470 (2010).

15. Bakker, F. L., Slachter, A., Adam, J-P. \& van Wees, B. J. Interplay of Peltier and Seebeck effects in nanoscale nonlocal spin valves. Phys. Rev. Lett. (in the press); preprint at http://arxiv.org/abs/1004.0118v1.

16. Hatami, M., Bauer, G. E. W., Zhang, Q. \& Kelly, P. J. Thermal spin-transfer torque in magnetoelectronic devices. Phys. Rev. Lett. 99, 066603 (2007).

17. Barnard, R. D. Thermoelectricity in Metals and Alloys (Taylor and Francis Ltd, 1972).

18. Tserkovnyak, Y., Brataas, A., Bauer, G. E. W. \& Halperin, B. I. Nonlocal magnetization dynamics in ferromagnetic heterostructures. Rev. Mod. Phys. 77, 1375-1421 (2005)

19. Gravier, L., Serrano-Guisan, S., Reuse, F. \& Ansermet, J. P. Thermodynamic description of heat and spin transport in magnetic nanostructures. Phys. Rev. B 73, 024419 (2006).

20. Dubi, Y. \& Di Ventra, M. Thermospin effects in a quantum dot connected to ferromagnetic leads. Phys. Rev. B 79, 081302 (2009).

21. Mott, N. F. \& Jones, H. The Theory of the Properties of Metals and Alloys (Oxford Univ. Press, 1936).

22. Brataas, A., Bauer, G. E. W. \& Kelly, P. J. Non-collinear magnetoelectronics. Phys. Rep. 427, 157-255 (2006).

23. Uchida, K. et al. Phenomenological analysis for spin-Seebeck effect in metallic magnets. J. Appl. Phys. 105, 07C908 (2009).

24. Valet, T. \& Fert, A. Theory of the perpendicular magnetoresistance in magnetic multilayers. Phys. Rev. B 48, 7099-7113 (1993).

25. van Son, P. C., van Kempen, H. \& Wyder, P. Boundary resistance of the ferromagnetic-nonferromagnetic metal interface. Phys. Rev. Lett. 58, 2271-2273 (1987).

26. Schmidt, G., Ferrand, D., Molenkamp, L. W., Filip, A. T. \& van Wees, B. J. Fundamental obstacle for electrical spin injection from a ferromagnetic metal into a diffusive semiconductor. Phys. Rev. B 62, R4790-R4793 (2000).

27. Vlaminck, V. \& Bailleul, M. Current-induced spin-wave Doppler shift. Science 322, 410-413 (2008).

28. Xiao, J., Bauer, G. E. W., Uchida, K., Saitoh, E. \& Maekawa, M. Theory of magnon-driven spin Seebeck effect. Phys. Rev. B 81, 214418 (2010).

29. Yu, H., Granville, S., Yu, D. P. \& Ansermet, J-Ph. Evidence for thermal spin transfer torque. Phys. Rev. Lett. 105, 146601 (2010).

30. Kittel, C. Introduction to Solid State Physics (John Wiley, 1996).

\section{Acknowledgements}

We would like to acknowledge J. G. Holstein, B. Wolfs and S. Bakker for technical assistance and T. Banarjee, I. J. Vera Marun, P. J. Zomer and S. Denega for critically reading the manuscript. This work was financed by the European EC Contracts IST-033749 'DynaMax', the 'Stichting voor Fundamenteel Onderzoek der Materie' (FOM) and NanoNed.

\section{Author contributions}

A.S. and B.J.v.W. conceived and designed the experiments. A.S. and F.L.B. carried out the main experiments and J-P.A. carried out some check-up experiments. All authors were involved in the analysis. A.S. wrote the paper, with the help of the co-authors.

\section{Additional information}

The authors declare no competing financial interests. Supplementary information accompanies this paper on www.nature.com/naturephysics. Reprints and permissions information is available online at http://npg.nature.com/reprintsandpermissions. Correspondence and requests for materials should be addressed to A.S. 